\title{
Methyltransferase-like 1 regulates lung adenocarcinoma A549 cell proliferation and autophagy via the AKT/mTORC1 signaling pathway
}

\author{
CHEN WANG, WEI WANG, XIAODAN HAN, LONGXIA DU, AILI LI and GUOJIN HUANG
}

Laboratory of Respiratory Diseases, The Affiliated Hospital of Guilin Medical University, Guilin, Guangxi 541001, P.R. China

Received August 13, 2020; Accepted November 13, 2020

DOI: 10.3892/ol.2021.12591

\begin{abstract}
Methyltransferase-like 1 (METTL1) is a transfer RNA and microRNA modifying enzyme. However, its role in lung adenocarcinoma (LUAD) remains unknown. The present study aimed to investigate the effect of METTL1 in LUAD and determine the association between METTL1 expression and prognosis of patients with LUAD. The expression profile of METTL1 in LUAD tissues was downloaded from public cancer databases and analyzed using the Gene Expression Profiling Interactive Analysis database and UALCAN online software. In addition, the association between METTL1 expression and prognosis of patients with LUAD was assessed using the Kaplan-Meier Plotter software. The effect of METTL1 in the A549 cell line was determined in vitro via overexpression and knockdown experiments. The results demonstrated that METTL1 was upregulated in LUAD tissues, and its increased expression was associated with unfavorable prognosis. Furthermore, METTL1 promoted proliferation and colony formation of A549 cells, and inhibited autophagy via the AKT/mechanistic target of rapamycin complex 1 signaling pathway. Taken together, the results of the present study suggest that METTL1 acts as an oncogene in LUAD, thus may be a potential prognostic predictor and therapeutic target for LUAD.
\end{abstract}

\section{Introduction}

Lung cancer is one of the most common types of cancer worldwide (1). The incidence and mortality rates of lung cancer in the United States in 2019 are estimated to be 13 and $23.5 \%$, respectively (2). The relative 5-year survival rate of lung cancer was $19 \%$ in the United States between 2008-2014 (1). The onset of lung cancer has been associated with genetic and

Correspondence to: Dr Guojin Huang, Laboratory of Respiratory Diseases, The Affiliated Hospital of Guilin Medical University, 15 Lequn Road, Guilin, Guangxi 541001, P.R. China

E-mail: hgjj@163.com

Key words: methyltransferase-like 1, lung adenocarcinoma, proliferation, autophagy, A549 cells epigenetic abnormalities (1). Although significant progress has been made during the last few decades, the molecular mechanisms underlying the development and progression of lung cancer remain partially unknown (1). Thus, identifying novel therapeutic targets for lung cancer is urgently required to provide personalized therapy to patients with specific molecular subtypes.

Autophagy is an important cellular process, where the intracellular damaged organelles and misfolded proteins are degraded and reused for cell metabolism, thus maintaining cellular, tissue and organismal homeostasis (3). mTOR is a negative regulator of autophagy, which is activated in response to both nutrients and growth factors (4). Furthermore, AKT links receptor tyrosine kinases to mechanistic target of rapamycin complex 1 (mTORC1), thereby repressing autophagy in response to insulin-like and other growth factor signals (4). Autophagy has dual roles in cancer. On the one hand, autophagy plays a significant role in tumor suppression; however, it also promotes cancer cell survival during metabolic stress (5).

Increasing evidence has suggested that RNA post-synthesis modifications control RNA folding, stability and function (6). In total, $>100$ different types of post-synthesis modifications have been identified on RNA, including transfer RNA (tRNA), ribosomal RNA (rRNA), messenger RNA (mRNA) and microRNA (miRNA) (6). All four RNA bases, as well as the ribose sugar, can be the targets of a variety of enzymes, such as methyltransferase-like 3, transfer RNA methyltransferase 10 and DNA methyltransferase like 2 (6). Dysregulation of specific RNA modifications has been associated with cancer (6). Recently, it has been reported that fat mass and obesity-associated protein, a demethylase of $\mathrm{N}^{6}$-methyladenosine $\left(\mathrm{m}^{6} \mathrm{~A}\right)$ of RNA, upregulates the Unc-51-like kinase 1 (ULK1), a critical molecule of autophagy, thus promoting the initiation of autophagy in HeLa and HEK293 cells (7). However, whether other types of RNA modification-related proteins affect autophagy remains unknown.

Methyltransferase-like 1 (METTL1) is a tRNA and miRNA modification enzyme, which forms a complex with WD repeat domain 4 to catalyze the 7-methylguanosine $\left(\mathrm{m}^{7} \mathrm{G}\right)$ modification of tRNA and miRNA in mammalian cells $(8,9)$. Previous studies have demonstrated that METTL1 is phosphorylated at Ser27 and inactivated by AKT and ribosomal S6 kinase (S6K) (10), and serves a positive role in murine embryonic stem 
cell proliferation (8). A study revealed that silencing METTL1 increases sensitivity of HeLa cells to 5-fluorouracil (11). Furthermore, METTL1 promotes miRNA processing in A549 cells to suppress cell migration (9), whereas its overexpression promotes hepatocellular carcinoma (HCC) and is associated with poor prognosis (12).

In the present study, METTL1 expression was assessed in LUAD tissues and its levels were associated with the prognosis of patients with LUAD. In addition, the molecular mechanisms underlying the effects of METTL1 were also investigated.

\section{Materials and methods}

Plasmids and small interfering (si)RNAs. The METTL1 coding sequence (NM_005371) fused with FLAG coding sequence at 3'-terminal was synthesized by Shanghai ShineGene Molecular Biotech, Inc., and sub-cloned into the pFLAG-CMV-4 vector, which was kindly provided by Dr Xianqiong Zou (Guilin Medical University). The METTL1 siRNA sequence (si-METTL1, 5'-GGACAUCUAGGCACC UCAATT-3') and control siRNA sequence (si-control, 5'-UU GAGGUGCCUAGAUGUCCTT-3') were synthesized by Shanghai GenePharma Co., Ltd.

Cell culture and transfection. The LUAD cell lines, A549 and H1993 were purchased from the Kunming Cell Bank of the Chinese Academy of Sciences. Cells were cultured and transfected as previously described (13). Briefly, cells were cultured in RPMI-1640 medium (Thermo Fisher Scientific, Inc.) supplemented with $10 \%$ fetal bovine serum (Thermo Fisher Scientific, Inc.) at $37^{\circ} \mathrm{C}$ in a humidified atmosphere of $5 \% \mathrm{CO}_{2}$.

A549 and H1993 cells were transfected at $37^{\circ} \mathrm{C}$ for $48 \mathrm{~h}$ using Lipofectamine ${ }^{\circledR} 3000$ reagent (Thermo Fisher Scientific, Inc.), according to the manufacturer's instructions. For the overexpression experiments, cells were transfected with $1 \mu \mathrm{g}$ of METTL1 overexpression plasmid or pFLAG-CMV-4 empty vector. For the METTL1 knockdown experiments, A549 and H1993 cells were transfected with $0.25 \mathrm{nmol}$ si-METTL1 or si-control. Cells were harvested for subsequent experimentation $48 \mathrm{~h}$ post-transfection.

Autophagy assays. A549 cells were transfected at $37^{\circ} \mathrm{C}$ for $48 \mathrm{~h}$, and then cells were harvested to detect autophagy markers LC3 and p62/sequestosome 1 (SQSTM1) using western blot (14). Autophagy inhibitor bafilomycin A1 (cat. no. tlrl-baf1, InvivoGen) was added into culture media (final concentration $100 \mathrm{nM}$ ) and incubated at $37^{\circ} \mathrm{C}$ for $1 \mathrm{~h}$ prior to harvesting cells in autophagy inhibition experiment. GFP-LC3 stable transformant HCC827 cells were transiently transfected with si-METTL1 or control siRNA for $48 \mathrm{~h}$. The cells were fixed with $2 \%$ paraformaldehyde at room temperature for $10 \mathrm{~min}$, followed by washing with PBS three times. Cells were observed under a fluorescent microscope (magnification, x400, Zeiss Axio Imager Z2, Carl Zeiss Microscopy, LLC), and the GFP-LC3 fluorescent puncta (autophagosome) were counted manually (14).

Western blotting. Western blotting was performed as previously described (15). Briefly, A549 and H1993 cells were harvested and lysed with lysis buffer (cat. no. R0020; Beijing Solarbio Science \& Technology Co., Ltd.) on ice for $5 \mathrm{~min}$. Subsequently, the lysate was centrifuged at $13,500 \mathrm{x} \mathrm{g}$ for $15 \mathrm{~min}$ at $4^{\circ} \mathrm{C}$ to obtain the supernatant. Protein concentration was measured using a BCA kit (cat. no. P0012, Beyotime Institute of Biotechnology), according to the manufacturer's instructions. Proteins $(30 \mu \mathrm{g})$ were separated by 12 or $15 \%$ SDS-PAGE gel, transferred onto polyvinylidene fluoride membranes and blocked with 5\% non-fat milk at room temperature for $2 \mathrm{~h}$. The membranes were incubated with the following primary antibodies: Rabbit anti-METTL1 antibody (cat. no. 14994-1; 1:2,000; ProteinTech Group, Inc.), rabbit anti-light chain (LC) 3B polyclonal antibody (cat. no. NB100-2220; 1:1,000; Novus Biologicals, LLC), rabbit anti-p62 polyclonal antibody (cat. no. 39749; 1:1,000), anti-AKT (cat. no. 9272; 1:1,000), anti-phosphorylated (p)-AKT (cat. no. 4060; 1:1,000), anti-S6K (cat. no. 9234; 1:1,000) and anti-p-S6K (cat. no. 9204; 1:1,000), overnight at $4^{\circ} \mathrm{C}$ (all purchased from Cell Signaling Technology, Inc). Following the primary incubation, membranes were incubated with secondary antibodies (horseradish peroxidase-conjugated goat anti-rabbit immunoglobulin G (IgG; cat. no. G-21234; 1:5,000) or goat anti-mouse IgG (cat. no. G-21040; 1:5,000) at room temperature for $1 \mathrm{~h}$ (both purchased from Pierce; Thermo Fisher Scientific, Inc.). Protein bands were developed using the enhanced chemiluminescence kit (cat. no. P0018; Beyotime Institute of Biotechnology).

Cell proliferation assay. Cell proliferation was determined via the Cell Counting Kit-8 (CCK-8; cat. no. CK04; Dojindo Molecular Technologies, Inc.) assay, according to the manufacturer's instructions. Briefly, A549 cells were seeded into 96-well plates at a density of $3 \times 10^{3}$ cells/well. Subsequently, $10 \mu 1$ CCK-8 solution was added to each well at $0,24,48,72$ and $96 \mathrm{~h}$, and cells were incubated at $37^{\circ} \mathrm{C}$ for $1 \mathrm{~h}$. The optical density (OD) values were analyzed at a wavelength of $450 \mathrm{~nm}$, using a microplate reader (EPOCH; BioTek Instruments, Inc.).

Colony formation assay. Cells were seeded into 6-well plates (600 cells/well for overexpression in A549, and 800 cells/well for other experiments), and $2 \mathrm{ml}$ RPMI-1640 medium supplemented with $10 \%$ fetal bovine serum (both purchased from Thermo Fisher Scientific, Inc.) was added into each well. Cells were cultured for $10-15$ days at $37^{\circ} \mathrm{C}$ in a humidified atmosphere of $5 \% \mathrm{CO}_{2}$. Following incubation, cells were washed three times with PBS and fixed with $4 \%$ neutral paraformaldehyde solution at room temperature for $30 \mathrm{~min}$. Cells were re-washed three times with PBS and $2 \mathrm{ml}$ of $1 \%$ crystal violet solution was added into each well at room temperature for an additional $2 \mathrm{~h}$. Cells were washed three times with PBS, the plates were dried and scanned with Epson Perfection V370 Photo scanner. Cell colonies were counted manually.

METTL1 expression in LUAD tissues and prognosis analysis. The METTL1 expression profiles in LUAD tissues and normal tissues were obtained from The Cancer Genome Atlas (TCGA, https://portal.gdc.cancer.gov) dataset and GSE10072 dataset in the Gene Expression Omnibus (GEO) database (https://www. ncbi.nlm.nih.gov/geo/query/acc.cgi?acc=GSE10072) (16). The TCGA LUAD results were analyzed using the online software 
Gene Expression Profiling Interactive Analysis (GEPIA; http://gepia.cancer-pku.cn). The METTL1 expression profiles in LUAD tissues and normal tissues of the GSE10072 dataset were analyzed using Prism 5.01 software (GraphPad Software, Inc.). METTL1 protein expression at in LUAD tissues was assessed using via immunohistochemical staining results downloaded from The Human Protein Atlas (https://www. proteinatlas.org). Survival analysis of patients with LUAD was performed using the Kaplan-Meier Plotter online software (http://kmplot.com). The log-rank test was used to determine survival probability. In addition, the expression of METTL1 at different stages was assessed using the UALCAN database (http://ualcan.path.uab.edu).

TCGA dataset and Gene Set Enrichment Analysis (GSEA). The TCGA-LUAD gene expression dataset (TCGA-LUAD. htseq_fpkm.tsv) was downloaded from the Xenahubs database (https://gdc.xenahubs.net/download/TCGA-LUAD. htseq_fpkm.tsv.gz). Tumor samples were classified into high- and low-METTL1 groups using the median METTL1 expression value as the cut-off. GSEA was performed using GSEA 4.0.3 software (http://www.broad.mit.edu/gsea), which was applied with the predefined gene sets. The number of permutations was set at 1,000 , and a gene set was considered significantly enriched with a False Discovery Rate score $<0.25$.

Functional analysis of GSE112180 dataset. The top 250 differentially expressed genes from the GEO dataset GSE112180 were identified by comparing the shCTRL and shMETTL1 groups using GEO2R (https://www.ncbi.nlm.nih. gov/geo/geo2r/?acc=GSE112180). The identified genes were uploaded onto the Database for Annotation, Visualization and Integrated Discovery (DAVID, https://david.ncifcrf.gov/tools. jsp). The genes were analyzed using functional annotation tool of DAVID to determine their association with signaling pathways. The related pathways were graphed using the gene counts (17).

Statistical analysis. Statistical analysis was performed using GraphPad Prism 5 software (GraphPad Software, Inc.) and SPSS 19.0 software (IBM Corp.). All experiments were performed independently at least three times and data are presented as the mean \pm standard deviation. Unpaired Student's t-test was used to compare differences between two groups, while one-way ANOVA followed by Tukey's post hoc test were used to compare differences between multiple groups. The log-rank test was used to determine survival probability. The $\chi^{2}$ test was used to assess the association between METTL1 expression and the clinicopathological characteristics of patients with LUAD. $\mathrm{P}<0.05$ was considered to indicate a statistically significant difference.

\section{Results}

METTL1 expression is upregulated in LUAD tissues, and its high expression is associated with unfavorable prognosis. The TCGA-LUAD dataset was analyzed using GEPIA. The results demonstrated that METTL1 expression was significantly upregulated in LUAD tissues $(\mathrm{n}=483)$ compared with normal lung tissues $(\mathrm{n}=59)(\mathrm{P}<0.05$; Fig. 1A). In addition, METTL1 expression was significantly higher in LUAD samples at different clinical stages compared with those in normal lung tissues $(\mathrm{P}<0.001$; Fig. 1B). The expression of METTL1 in LUAD samples was also confirmed using data from another study (GSE10072) in the Gene Expression Omnibus (GEO) database (16) (Fig. 1C and D). As METTL1 expression from the TCGA-LUAD and GSE10072 datasets was at RNA level, its expression at protein level was further assessed via immunohistochemistry staining, using the Human Protein Atlas database. The results demonstrated that there were five LUAD samples with METTL1 positive staining (2 medium-, and 3 low-staining samples) among nine assessed lung cancer samples, which confirmed that METTL1 protein expression is upregulated in LUAD (Fig. 1E).

To determine the association between METTL1 expression and the clinicopathological characteristics of patients with LUAD, patients in the TCGA-LUAD dataset (Please note: the value of some characteristics was absent in the original TCGA LUAD dataset, so the number of patients for different characteristics varies) were classified into METTL1 low or high groups, with the median METTL1 expression value set as the cut-off. The $\chi^{2}$ test was used to assess the association between METTL1 expression and the clinicopathological characteristics of patients with LUAD, and the results demonstrated that METTL1 was only associated with the M stage (M1 vs. M0; $\mathrm{P}<0.01$; Table I).

The clinical value of METTL1 on disease prognosis was assessed by analyzing the overall survival (OS), first progression (FP) and disease-free survival (DFS) of patients with LUAD, using the Kaplan-Meier Plotter software. Patients were divided into METTL1-high and METTL1-low groups, with the median METTL1 mRNA value set as the cut-off. The results demonstrated that patients in the METTL1-low group had improved OS and FP rates compared with those in the METTL1-high group (Fig. 1F and G; P<0.0001). However, no significant difference was observed in DFS between the two groups (data not shown). The Cox regression model was used to perform multivariate analysis of METTL1 expression on OS and FP. The results demonstrated that METTL1 expression was an independent indicator for OS, but not for FP (Table II). In addition, AJCC stage $\mathrm{T}$ was an independent indicator for OS and FP, and AJCC stage $\mathrm{N}$ was an independent indicator for OS only (Table II) (18).

METTL1 promotes colony formation and A549 cell proliferation. To investigate the role of METTL1 in vitro, western blot analysis was performed to detect METTL1 protein expression in the A549 lung cancer cell line. As presented in Fig. 2A, METTL1 was upregulated in A549 cells compared with BEAS-2B normal lung epithelial cells. Thus, the A549 cell line was used for subsequent experiments. A549 cells were subsequently transfected with METTL1 plasmids or si-METTL1 for METTL1 overexpression and silencing experiments, respectively. Western blot analysis demonstrated that METTL1 protein expression increased and decreased in the overexpression and knockdown experiments, respectively (Fig. 2B). The effect of METTL1 overexpression and silencing in A549 cell proliferation and colony formation was investigated. The results demonstrated that A549 cell proliferation increased following METTL1 overexpression, the effects of which were attenuated following transfection with si-METTL1 
A

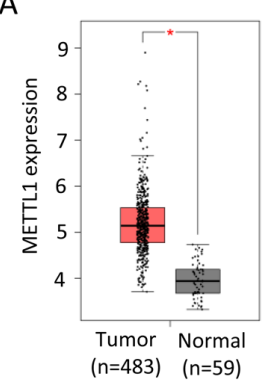

B

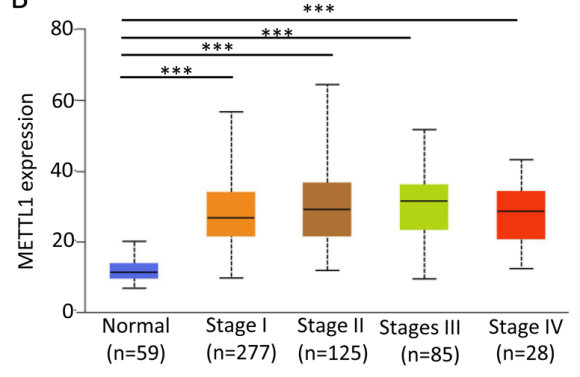

C

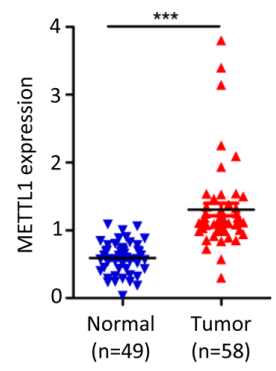

D
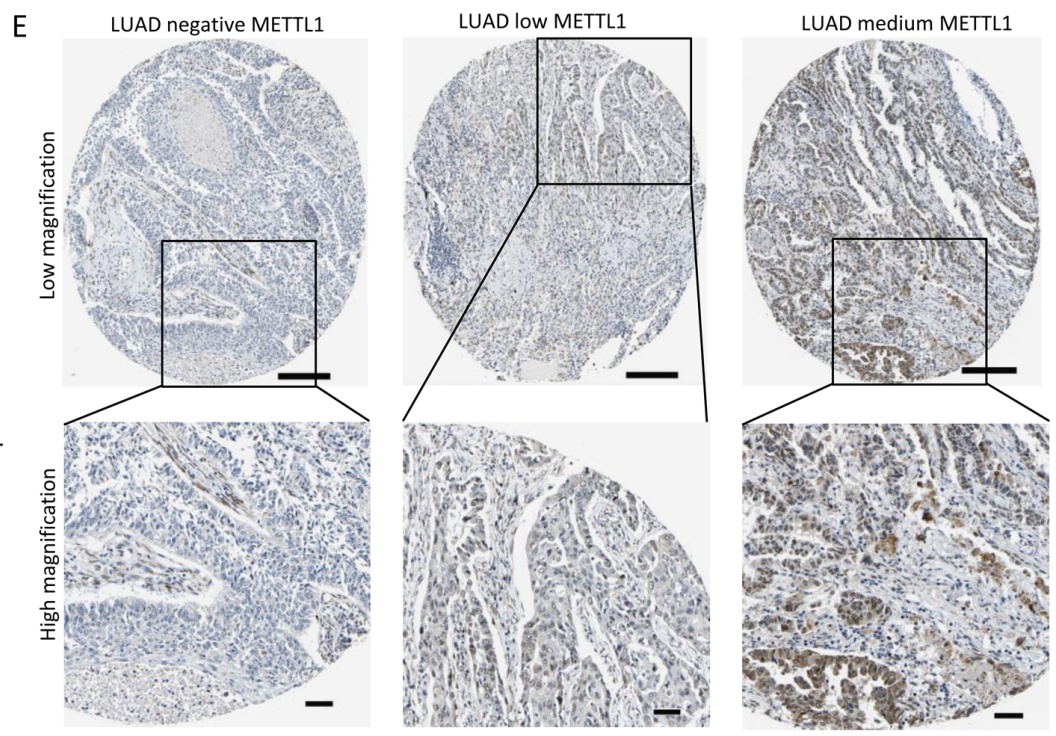

$\mathrm{F}$

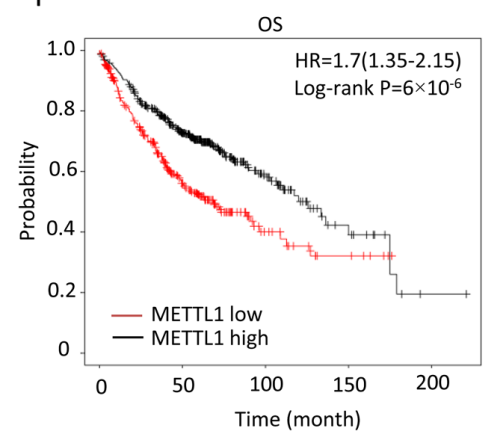

G

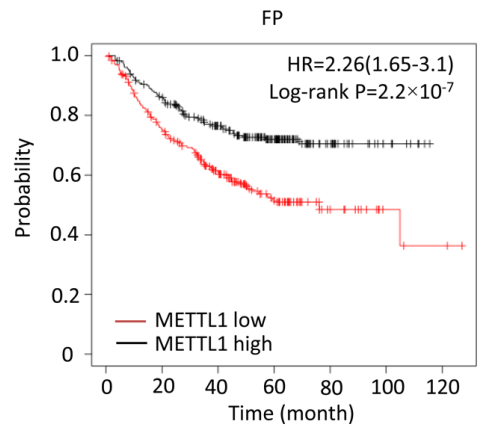

Figure 1. METTL1 expression is upregulated in LUAD, and its increased expression is associated with unfavorable OS and FP. (A) METTL1 expression profile in LUAD tissues $(n=483)$ and normal lung tissues $(n=59)$ from TCGA database was analyzed using Gene Expression Profiling Interactive Analysis software. METTL1 expression was significantly upregulated in LUAD tissues. (B) METTL1 expression was significantly higher in different LUAD stages compared with normal tissues. No significant differences were observed among the different tumor stages. The METTL1 expression profile in different LUAD stages (stage I, $n=277$; stage II, $n=125$; stage III, $n=85$ and stage IV, $n=28$ ) and normal lung tissues ( $n=59)$ from TCGA database was analyzed using UALCAN software. (C) The METTL1 expression profile in LUAD tissues $(n=58)$ and normal lung tissues $(n=49)$ from the GSE10072 dataset in the GEO database was analyzed using GraphPad Prism 5.0 software. METTL1 expression was significantly upregulated in LUAD tissues. (D) METTL1 expression was significantly higher in different LUAD stages compared with normal tissues. No significant differences were observed among the different stages. The METTL1 expression profile in different stages (stage I, $n=22$; stage II, $n=21$; stage III, n=12 and stage IV, $n=3$ ) and normal lung tissues ( $n=49$ ) from the GSE10072 dataset in the GEO database was analyzed using GraphPad Prism 5.0 software. (E) METTL1 expression was upregulated in LUAD tissues. Representative images of immunohistochemistry staining from the Human Protein Atlas database. Scale bar at low magnification, $200 \mu \mathrm{M}$; high magnification, $50 \mu \mathrm{M}$. HPA Patients' ID, negative: 2268, medium: 3052, low: 2403. (F) High METTL1 expression was associated with unfavorable OS. The OS curve of 719 patients was plotted [cut-off value, 347; HR=1.7 (1.35-2.15), 95\% CI; log-rank $\left.\mathrm{P}=6 \times 10^{-6}-05\right]$. (G) High METTL1 expression was associated with unfavorable FP. The FP curve of 461 patients was plotted [cut-off value, 324; HR=2.26 (1.65-3.1), 95\% CI; log-rank P=2.2x10-7]. The online Kaplan Meier Plotter software was used to construct the OS and FP graphs. "P<0.05, ${ }^{* * * *} \mathrm{P}<0.001$. METTL1, methyltransferase-like 1; LUAD, lung adenocarcinoma; OS, overall survival; FP, first progression; TCGA, The Cancer Genome Atlas; GEO, Gene Expression Omnibus; HR, hazard ratio; CI, confidence interval.

(Fig. 2C). In addition, METTL1 overexpression increased the colony count, which decreased in METTL1-silenced A549 cells (Fig. 2D). The effects of METTL1 on cell proliferation and colony formation were further confirmed in LUAD H1993 cells by overexpressing METTL1 (Fig. 2C and D).
METTL1 inhibits autophagy in A549 cells. It is well-known that LC3B conversion (LC3-I to LC3-II) and lysosomal degradation of LC3-II reflect the progression of autophagy. In addition, it has been reported that $\mathrm{p} 62$ /sequestosome 1 (SQSTM1) protein is degraded by autophagy (14). Thus, 
Table I. Association between METTL1 expression and the clinicopathological characteristics of patients with lung adenocarcinoma $(\mathrm{n}=517)$.

\begin{tabular}{|c|c|c|c|c|c|c|}
\hline \multirow[b]{2}{*}{ Characteristics } & \multirow[b]{2}{*}{ Variables } & \multirow[b]{2}{*}{ Number of patients, $n$} & \multicolumn{2}{|c|}{$\begin{array}{l}\text { METTL1 } \\
\text { expression }\end{array}$} & \multirow[b]{2}{*}{$\chi^{2}$} & \multirow[b]{2}{*}{ P-value } \\
\hline & & & Low, $\mathrm{n}$ & High, $\mathrm{n}$ & & \\
\hline \multirow[t]{2}{*}{ Age, years } & $<60$ & 137 & 69 & 68 & 0.0335 & 0.8549 \\
\hline & $\geq 60$ & 362 & 179 & 183 & & \\
\hline \multirow[t]{2}{*}{ Sex } & Female & 277 & 140 & 137 & 0.0972 & 0.7552 \\
\hline & Male & 240 & 118 & 122 & & \\
\hline \multirow[t]{2}{*}{$\mathrm{T}$} & $\mathrm{T} 1$ & 168 & 90 & 78 & 1.3390 & 0.2471 \\
\hline & $\mathrm{T} 2 / 3 / 4$ & 349 & 168 & 181 & & \\
\hline \multirow[t]{2}{*}{$\mathrm{N}$} & N0 & 332 & 169 & 163 & 0.5588 & 0.4547 \\
\hline & $\mathrm{N} 1 / 2 / 3$ & 173 & 82 & 91 & & \\
\hline \multirow[t]{2}{*}{ M } & M0 & 350 & 173 & 177 & 8.1040 & $0.0044^{\mathrm{a}}$ \\
\hline & M1 & 25 & 5 & 20 & & \\
\hline \multirow[t]{2}{*}{ Stage } & Stage 1 & 276 & 142 & 134 & 3.6700 & 0.0554 \\
\hline & Stage $2 / 3 / 4$ & 213 & 91 & 122 & & \\
\hline \multirow[t]{2}{*}{ DLCO predictive percent } & $<80$ & 124 & 68 & 56 & 0.8760 & 0.3493 \\
\hline & $\geq 80$ & 75 & 36 & 39 & & \\
\hline \multirow[t]{2}{*}{ Bronchondilator FEV 1\% } & $<80$ & 62 & 31 & 31 & 0.0501 & 0.8229 \\
\hline & $\geq 80$ & 79 & 38 & 41 & & \\
\hline \multirow[t]{2}{*}{ Location in lung parenchyma } & Central lung & 64 & 34 & 30 & 0.0023 & 0.9615 \\
\hline & Peripheral lung & 127 & 67 & 60 & & \\
\hline \multirow[t]{2}{*}{ Person neoplasm cancer status } & Tumor free & 311 & 156 & 155 & 0.1945 & 0.6592 \\
\hline & With tumor & 109 & 52 & 57 & & \\
\hline \multirow[t]{2}{*}{ Smoking history indicator } & $1 / 2$ & 197 & 96 & 101 & 0.1221 & 0.7268 \\
\hline & $3 / 4 / 5$ & 308 & 155 & 153 & & \\
\hline \multirow[t]{2}{*}{ Packs of cigarettes per year } & $<40$ & 175 & 86 & 89 & 0.1813 & 0.6702 \\
\hline & $\geq 40$ & 177 & 91 & 86 & & \\
\hline
\end{tabular}

${ }^{\mathrm{a}} \mathrm{P}<0.01$. METTL1, methyltransferase-like 1; TNM, tumor-node-metastasis; DLCO, diffusing capacity of the lungs for carbon monoxide.

LC3B and p62 are considered markers of autophagy (14). To assess the effects of METTL1 on autophagy, A549 cells were transfected with METTL1 overexpression plasmids at $37^{\circ} \mathrm{C}$ for $48 \mathrm{~h}$ and were subsequently treated with the autophagy inhibitor, bafilomycin $\mathrm{A} 1(100 \mathrm{nM})$ at $37^{\circ} \mathrm{C}$ for $1 \mathrm{~h}$. Cells were subsequently harvested, cell lysates were prepared and the protein expression levels of LC3B and p62 were determined in the lysates via western blot analysis. The results demonstrated that METTL1 overexpression suppressed the conversion of LC3-I to LC3-II, turnover of LC3-II and degradation of p62/SQSTM1. Furthermore, the inhibitory effect of METTL1 was enhanced following treatment with bafilomycin A1 (Fig. 3A), implying that METTL1 may inhibit autophagy. To confirm these findings, A549 cells were transfected with si-METTL1 at $37^{\circ} \mathrm{C}$ for $48 \mathrm{~h}$ and subsequently treated with $100 \mathrm{nM}$ bafilomycin $\mathrm{A} 1$ at $37^{\circ} \mathrm{C}$ for $1 \mathrm{~h}$. The protein expression levels of LC3B and p62/SQSTM1 was detected via western blot analysis. The results demonstrated that METTL1 knockdown promoted the conversion of LC3-I to LC3-II and degradation of p62/SQSTM1. LC3-II and p62/SQSTM1 levels accumulated following treatment with bafilomycin A1 in the si-METTL1 group compared with the control group (Fig. 3B), suggesting that METTL1 knockdown may enhance autophagy. To confirm the effects of METTL1 on autophagy, HCC827 cells stably expressing GFP-LC3 were transfected with si-METTL1 and fluorescent puncta (autophagosome) were observed under a fluorescent microscope and quantified. The results demonstrated that silencing METTL1 enhanced autophagy (Fig. 3). Taken together, the overexpression and silencing experiments indicated that METTL1 may inhibit autophagy.

METTL1 activates AKT/mTORC1 signaling. To determine the molecular mechanism underlying the effect of METTL1 on the inhibition of autophagy, GSEA was performed using the TCGA-LUAD dataset. The results demonstrated that the gene sets of the phosphatidylinositol 3-kinase (PI3K)/AKT/mTOR and MTORC1 signaling pathways were enriched in the high-METTL1 expression group (Fig. 4A and B). Furthermore, the GSE112180 dataset was analyzed using GEO2R, and the top 250 differentially 
Table II. Cox regression multivariate analysis of METTL1 on OS and FP of patients with lung adenocarcinoma.

\begin{tabular}{llll}
\hline & Characteristics & Hazard ratio (95\% CI) & P-value \\
\hline OS & Sex & $1.54(0.89-2.66)$ & 0.1270 \\
& Stage & $0.41(0.15-1.11)$ & 0.0795 \\
AJCC stage T & $2.09(1.12-3.89)$ & $0.0204^{\mathrm{a}}$ \\
AJCC stage N & $2.64(1.03-6.75)$ & $0.0434^{\mathrm{a}}$ \\
& Smoking history & $1.96(0.44-8.63)$ & 0.3700 \\
METTL1 & $2.07(1.24-3.46)$ & $0.0056^{\mathrm{b}}$ \\
FP & Sex & $1.33(0.71-2.48)$ & 0.3718 \\
& Stage & $1.37(0.14-13.89)$ & 0.7883 \\
AJCC stage T & $3.11(1.30-7.44)$ & $0.0109^{\mathrm{a}}$ \\
AJCC stage N & $2.14(0.21-21.41)$ & 0.5185 \\
& Smoking history & $1.51(0.73-3.13)$ & 0.2642 \\
METTL1 & $1.54(0.82-2.9)$ & 0.1836 \\
\hline
\end{tabular}

${ }^{\mathrm{a}} \mathrm{P}<0.05,{ }^{\mathrm{b}} \mathrm{P}<0.01$. METTL1, methyltransferase-like 1; OS, overall survival; FP, first progression; CI, confidential interval; AJCC, American Joint Committee on Cancer.

A

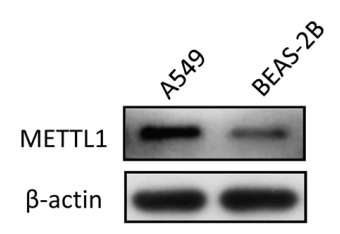

B

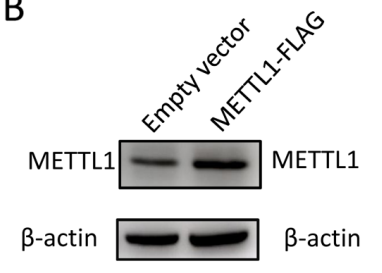

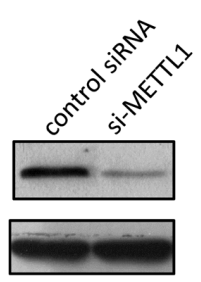

C

A549

A549

H1993
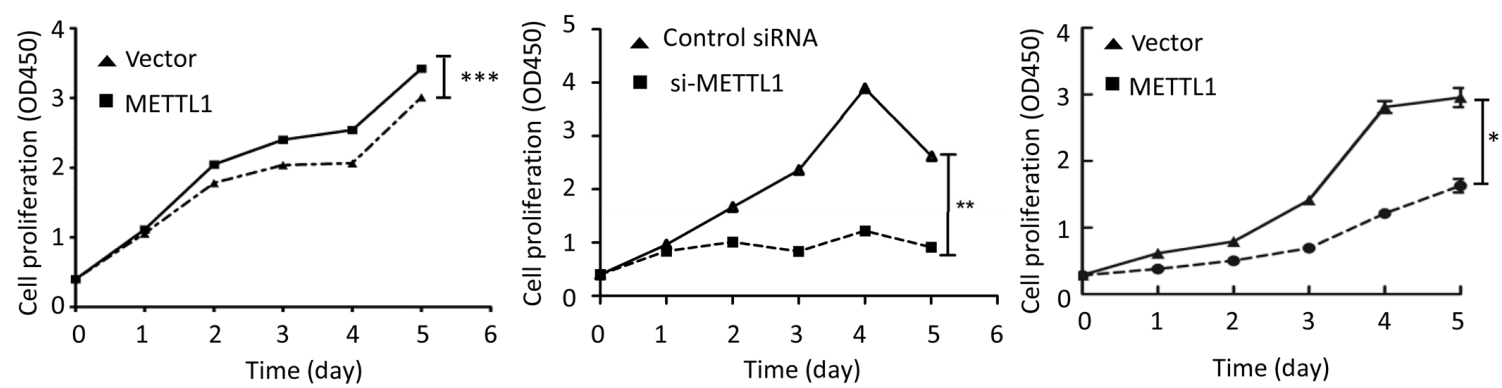

D

A549
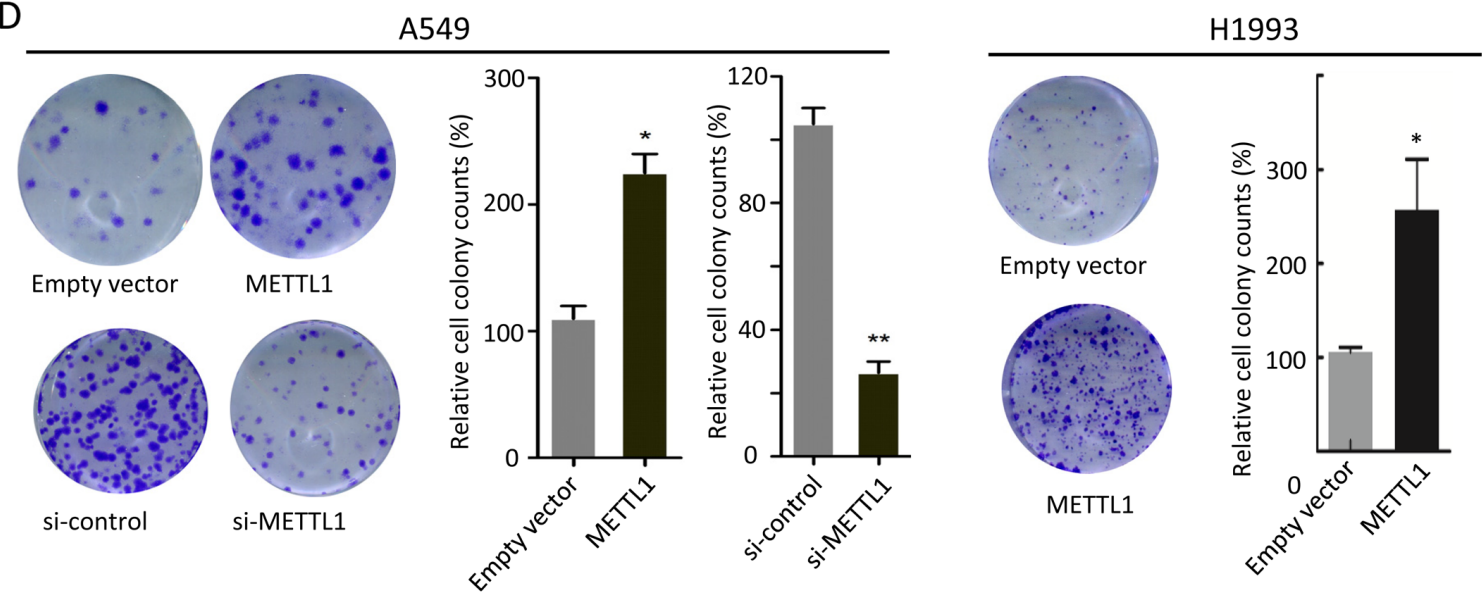

Figure 2. METTL1 promotes colony formation and A549 cell proliferation. (A) METTL1 expression was increased in A549 cells. (B) Western blot analysis demonstrated that METTL1 protein was upregulated and downregulated in the overexpression and knockdown experiments, respectively. Overexpression of METTL1 promoted A549 and H1993 cell (C) proliferation and (D) colony formation. METTL1 silencing attenuated A549 and H1993 cell (C) proliferation and (D) colony formation. $\mathrm{P}<0.05,{ }^{* *} \mathrm{P}<0.01,{ }^{* * *} \mathrm{P}<0.001$. METTL1, methyltransferase-like 1 ; si, small interfering; OD, optical density. 
A
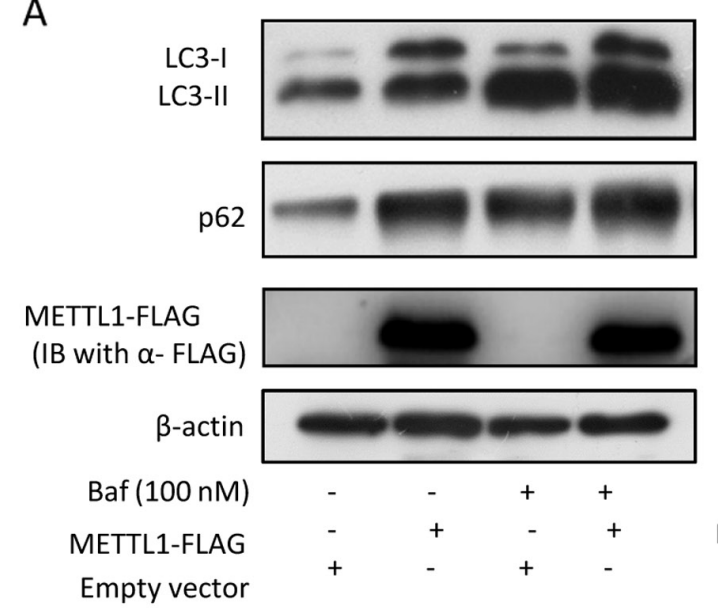

B

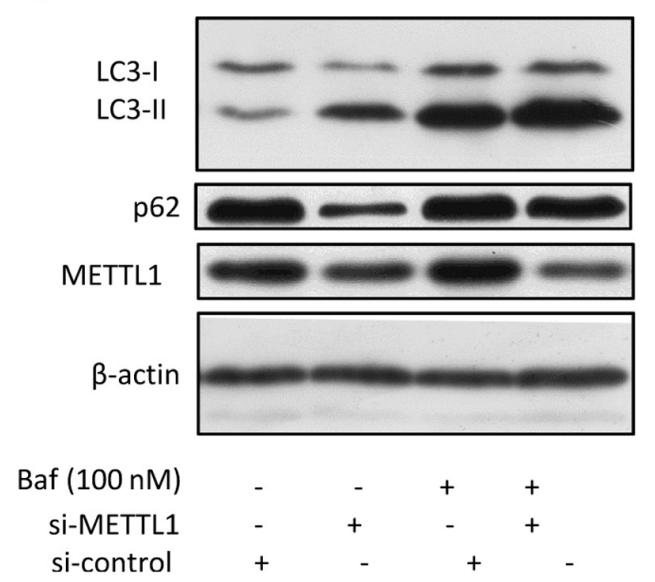

C

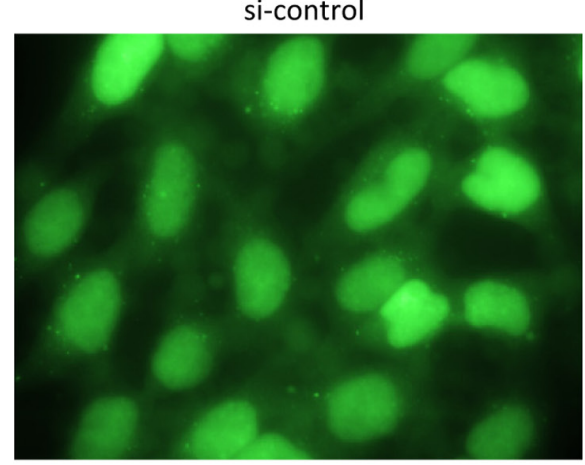

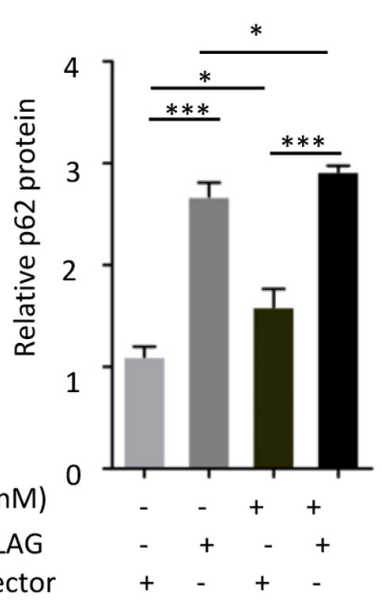

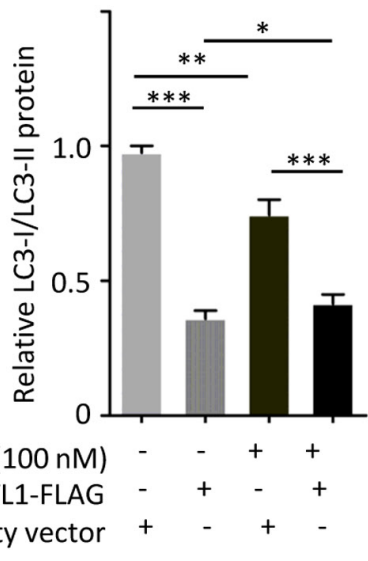

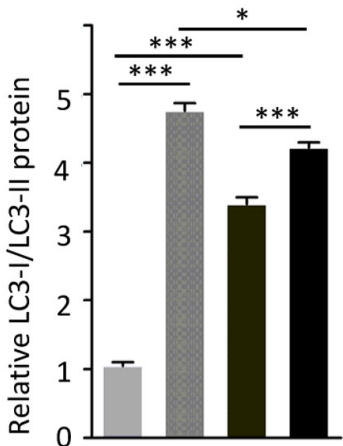

Baf $(100 n M) \quad-\quad+\quad+$ si-METTL1 - + - + si-control +-++

si-METTL1

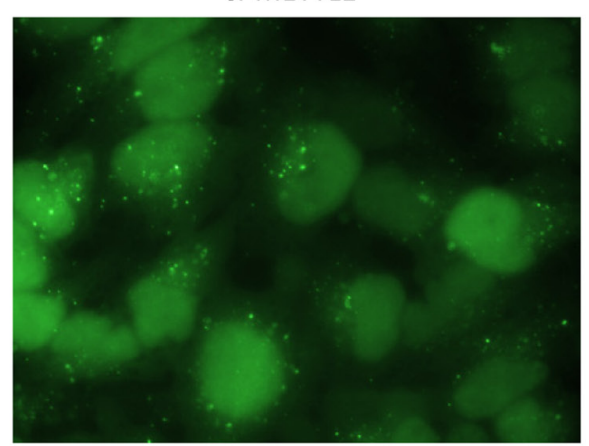

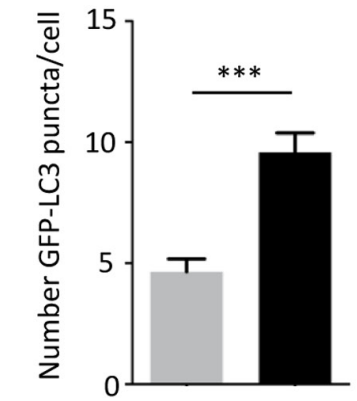

si-conrol si-METTL1

Figure 3. METTL1 inhibits autophagy in A549 cells. (A) METTL1 overexpression inhibited the conversion of LC3-I to LC3-II and the degradation of p62/SQSTM1, suggesting that METTL1 overexpression may inhibit autophagy. (B) METTL1 knockdown enhanced the conversion of LC3-I to LC3-II and the degradation of p62/SQSTM1, suggesting that METTL1 knockdown may promote autophagy. (C) METTL1 knockdown increased both the size and number of GFP-LC3 puncta in HCC827/GFP-LC3 cells, suggesting that METTL1 knockdown may promote autophagy. ${ }^{*} \mathrm{P}<0.05,{ }^{* * *} \mathrm{P}<0.01,{ }^{* * * *} \mathrm{P}<0.001 . \mathrm{METTL} 1$, methyltransferase-like 1; SQSTM1, sequestosome 1; si, small interfering.

expressed genes were identified. These genes were analyzed using the Database for Annotation, Visualization and Integrated Discovery (DAVID). The results revealed that PI3K/AKT/mTOR signaling was activated by METTL1 (Fig. 4C), which was consistent with the GSEA results. Subsequently, cellular experiments were performed to determine the effects of METTL1 on the aforementioned signaling pathways. A549 cells were transfected with METTL1 overexpression plasmids or si-METTL1 $37^{\circ} \mathrm{C}$ for $48 \mathrm{~h}$. Following transfection, the cells were collected and protein lysates were prepared and resolved via western blot analysis. METTL1 overexpression notably increased the expression levels of $\mathrm{p}-\mathrm{AKT}$ and $\mathrm{p}-\mathrm{S} 6 \mathrm{~K}$, while the total protein levels of AKT and S6K remained unchanged compared with those in the empty vector group. Conversely, knockdown of METTL1 downregulated p-AKT and p-S6K protein expression, while the total protein levels of AKT and S6K remained unchanged compared with those in the si-control group. Taken together, these results suggest that METTL1 may activate the AKT/mTORC1 signaling pathway. 
A

HALLMARK_MTORC1_SIGNALING

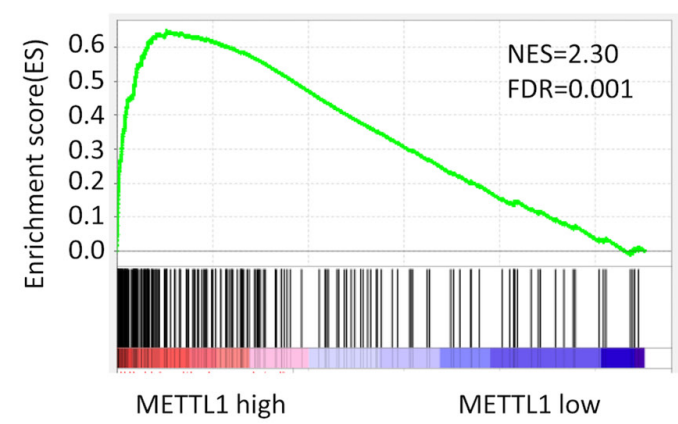

C

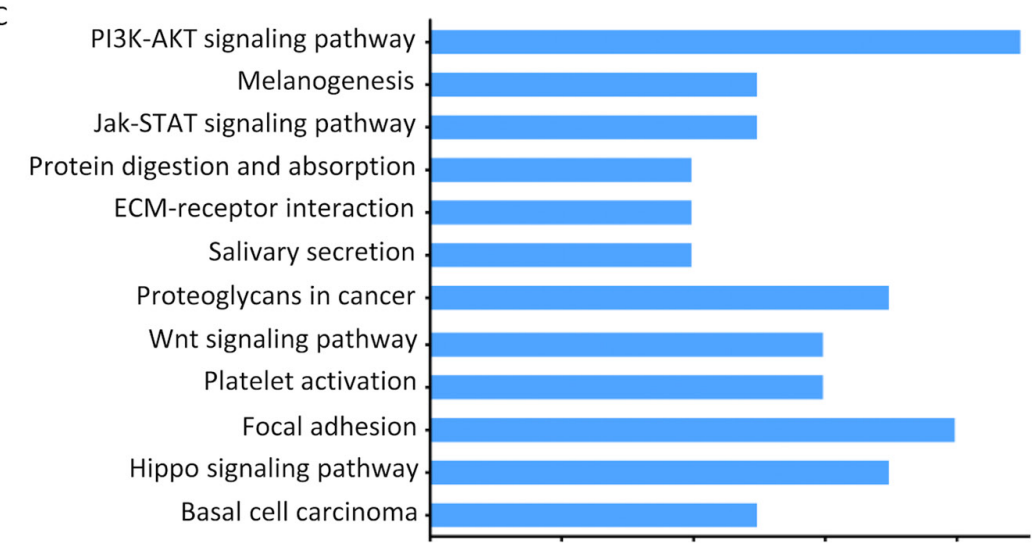

HALLMARK_PI3K_AKT_MTOR_SIGNALING

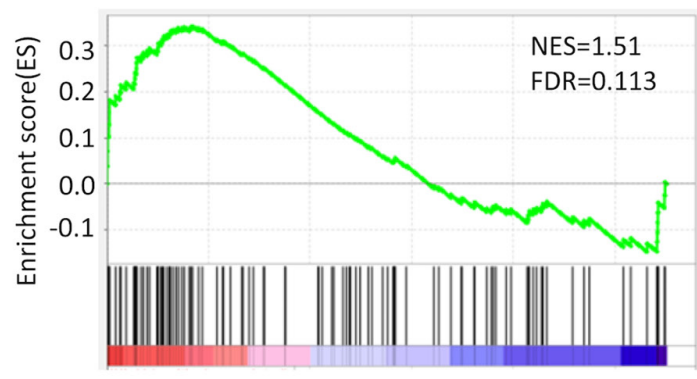

METTL1 high

METTL1 low

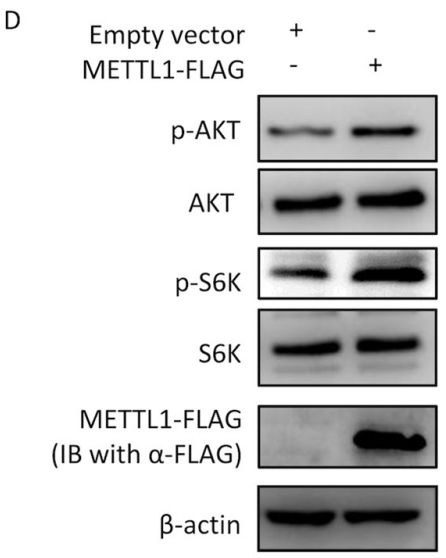

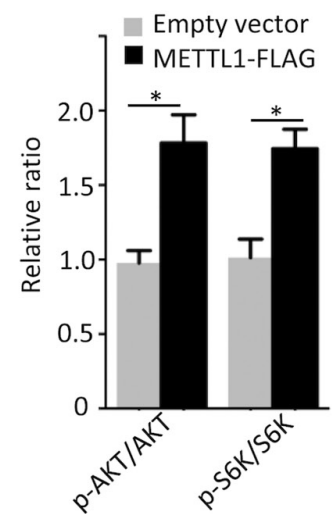

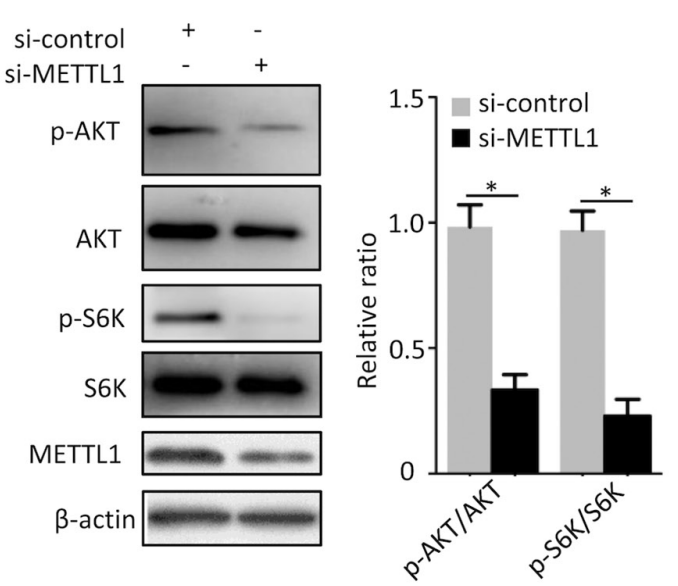

Figure 4. METTL1 activates the AKT/mTORC1 signaling pathway. GSEA analysis of the TCGA-LUAD dataset revealed that the gene sets (A) HALLMARK MTORC1_SIGNALING and (B) HALLMARK_PI3K_AKT_MTOR_SIGNALING were enriched in the high-METTL1 expression group. (C) DAVID analysis of the GSE112180 dataset indicated that the gene cluster of the PI3K/AKT signaling pathway was enriched in the high-METTL1 expression group (D) METTL1 overexpression increased the protein levels of p-AKT and p-S6K, the effects of which were reversed following METTL1 silencing. Total protein levels of AKT and S6K remained unchanged compared with the control group. "P<0.05. METTL1, methyltransferase-like 1; p-AKT, phosphorylated protein kinase B; mTORC1, mechanistic target of rapamycin complex 1; GSEA, Gene Set Enrichment Analysis; TCGA, The Cancer Genome Atlas; LUAD, lung adenocarcinoma; DAVID, Database for Annotation, Visualization and Integrated Discovery; PI3K, phosphatidylinositol 3-kinase; si, small interfering.

\section{Discussion}

It has been reported that METTL1 catalyzes the $\mathrm{m}^{7} \mathrm{G}$ modification of tRNA and miRNA $(8,9)$. Previous studies investigated the effect of METTL1 in different types of cancer cells; however, their findings were controversial $(12,18)$. Tian et al (12) demonstrated that METTL1 is upregulated in HCC at both the mRNA and protein levels, whereby increased levels are associated with poor outcomes. Furthermore, METTL1 was reported to promote
HCC cell proliferation and migration in vitro, suggesting that METTL1 acts as an oncogene in HCC. Conversely, Liu et al (19) reported that METTL1 is downregulated in colon cancer, thus acts as a tumor suppressor molecule. The present study investigated METTL1 expression in two different LUAD cohorts obtained from two databases. The results demonstrated that METTL1 was significantly upregulated in LUAD, which was consistent with the results on HCC (12). In addition, METTL1 expression did not significantly changed from LUAD stage I to 
stage IV, indicating that METTL1 upregulation occurs early during LUAD tumorigenesis. Taken together, these results suggest that METTL1 may be a potential diagnostic marker for LUAD. Although survival analysis demonstrated that high METTL1 expression was associated with unfavorable OS and FP, multivariate analysis indicated that METTL1 was an independent indicator for OS, but not for FP.

The present study demonstrated that knockdown of METTL1 in A549 cells inhibited cell proliferation and colony formation, whereas METTL1 overexpression in the same cell line had the opposite effects. These findings were in agreement with the aforementioned study on HCC (12). Autophagy is a lysosomal-related degradation pathway (3). In response to microenvironmental stimuli, cells can eliminate proteins and lipids through autophagy in order to maintain cellular homeostasis (3). Furthermore, autophagy may act as a tumor-suppressing mechanism during tumor initiation, or as a tumor-promoting process in established tumors (20). A study demonstrated that METTL1 exerts inhibitory effects on the phosphatase and tensin homolog (PTEN) signaling (12). Given that PTEN is considered a key inhibitory factor upstream mTORC1, which acts as a suppressor of autophagy, the present study hypothesized that METTL1 may play a role in the regulation of autophagy. Thus, the effects of METTL1 knockdown and overexpression on autophagy were investigated. The results demonstrated that METTL1 knockdown and overexpression enhanced and inhibited autophagy, respectively. To determine the molecular mechanisms underlying the effects of METTL1, bioinformatics analysis was performed followed by verification with in vitro experiments. The results demonstrated that overexpression of METTL1 promoted activation of the AKT/mTORC1 signaling pathway. AKT and mTORC1 serve a pivotal role in cell proliferation, and both proteins are hyperactivated in several types of cancer, such as lymphoma, breast cancer and lung cancer $(21,22)$. As expected, the results of the present study demonstrated that high METTL1 expression in LUAD was associated with unfavorable prognosis. Taken together, the present study provided cellular and mechanistic evidence to elucidate the role of METTL1 in LUAD tumorigenesis. To the best of our knowledge, the present study was the first to investigate the role of METTL1 in the regulation of autophagy and AKT/mTORC1 signaling. However, the in vitro experiments were only performed in the A549 cell line, thus, further experiments should be performed in more LUAD cell lines to confirm the findings presented here.

The results of the present study on LUAD and those of a previous study on HCC (12) suggest that METTL1 may acts as an oncogene. Thus, it is of great importance to address, in future studies, the molecular mechanism underlying the regulation of METTL1 expression, and how changes in METTL1 expression affect AKT/mTORC1 signaling and carcinogenesis. Since METTL1 promotes miRNA processing (9), certain miRNA(s) may be associated with METTL1 expression and AKT/mTORC1 signaling. Thus, it may be useful to identify the miRNA(s) involved in this process.

In summary, the present study demonstrated that METTL1 expression was upregulated in LUAD tissues, which was associated with unfavorable prognosis. Furthermore, METTL1 may act via the AKT/mTORC1 pathway in LUAD cells. Taken together, these results suggest that METTL1 acts as an onco- gene in LUAD and may be a potential diagnostic biomarker and therapeutic target for LUAD.

\section{Acknowledgements}

The authors would like to thank Dr Xianqiong Zou from Guilin Medical University (Guilin, China) for providing the pFLAG-CMV-4 plasmid.

\section{Funding}

The present study was supported in part by the Hundred Talents Program of Guangxi (to GH), the Natural Science Foundation of Guangxi (grant no. 2020JJA140139), the Research Enhancement Project for Junior Faculty in Higher Education Institutes of Guangxi (grant no. 2019KY0522), the Scientific Research Project for Junior Faculty in Guilin Medical College (grant no. 2018glmcy055) and the Guangxi Key Laboratory of Molecular Medicine in Liver Injury and Repair (grant nos. GXLIRMMKL-201802 and GXLIRMMKL-201816).

\section{Availability of data and materials}

All data generated or analyzed during the present study are included in this published article.

\section{Authors' contributions}

GH conceived the present study. CW, WW, XH, LD and AL performed the experiments. $\mathrm{GH}$ and $\mathrm{CW}$ confirmed the authenticity of all the raw data. $\mathrm{GH}$ and $\mathrm{CW}$ analyzed and interpreted the data. $\mathrm{CW}$ and $\mathrm{GH}$ drafted the initial manuscript. All authors have read and approved the final manuscript.

\section{Ethics approval and consent to participate}

Not applicable.

\section{Patient consent for publication}

Not applicable.

\section{Competing interests}

The authors declare that they have no competing interests.

\section{References}

1. Herbst RS, Morgensztern D and Boshoff C: The biology and management of non-small cell lung cancer. Nature 553: 446-454, 2018

2. Siegel RL, Miller KD and Jemal A: Cancer statistics, 2019. CA Cancer J Clin 69: 7-34, 2019.

3. Levine B and Kroemer G: Biological Functions of Autophagy Genes: A Disease Perspective. Cell 176: 11-42, 2019.

4. Levine B and Kroemer G: Autophagy in the pathogenesis of disease. Cell 132: 27-42, 2008.

5. White E: The role for autophagy in cancer. J Clin Invest 125: 42-46, 2015.

6. Barbieri I and Kouzarides T: Role of RNA modifications in cancer. Nat Rev Cancer 20: 303-322, 2020.

7. Jin S, Zhang X, Miao Y, Liang P, Zhu K, She Y, Wu Y, Liu DA, Huang J, Ren J, et al: $\mathrm{m}^{6} \mathrm{~A}$ RNA modification controls autophagy through upregulating ULK1 protein abundance. Cell Res 28: 955-957, 2018. 
8. Lin S, Liu Q, Lelyveld VS, Choe J, Szostak JW and Gregory RI Mettl1/Wdr4-Mediated $\mathrm{m}^{7} \mathrm{G}$ tRNA Methylome Is Required for Normal mRNA Translation and Embryonic Stem Cell Self-Renewal and Differentiation. Mol Cell 71: 244-255.e5, 2018.

9. Pandolfini L, Barbieri I, Bannister AJ, Hendrick A, Andrews B, Webster N, Murat P, Mach P, Brandi R, Robson SC, et al: METTL1 Promotes let-7 MicroRNA Processing via $\mathrm{m}^{7} \mathrm{G}$ Methylation. Mol Cell 74: 1278-1290.e9, 2019.

10. Cartlidge RA, Knebel A, Peggie M, Alexandrov A, Phizicky EM and Cohen P: The tRNA methylase METTL1 is phosphorylated and inactivated by PKB and RSK in vitro and in cells. EMBO J 24: 1696-1705, 2005.

11. Okamoto M, Fujiwara M,Hori M, Okada K, Yazama F, Konishi H, Xiao Y, Qi G, Shimamoto F, Ota T, et al: tRNA modifying enzymes, NSUN2 and METTL1, determine sensitivity to 5-fluorouracil in HeLa cells. PLoS Genet 10: e1004639, 2014.

12. Tian QH, Zhang MF, Zeng JS, Luo RG, Wen Y, Chen J, Gan LG and Xiong JP: METTL1 overexpression is correlated with poor prognosis and promotes hepatocellular carcinoma via PTEN. J Mol Med (Berl) 97: 1535-1545, 2019.

13. Wang Q, Li A, Jin J and Huang G: Targeted interfering DEP domain containing 1 protein induces apoptosis in A549 lung adenocarcinoma cells through the NF- $\mathrm{\kappa B}$ signaling pathway. Onco Targets Ther 10: 4443-4454, 2017.

14. Klionsky DJ, Abdelmohsen K, Abe A, Abedin MJ, Abeliovich H, Acevedo Arozena A, Adachi H, Adams CM, Adams PD, Adeli K, et al: Guidelines for the use and interpretation of assays for monitoring autophagy (3rd edition). Autophagy 12: 1-222, 2016.

15. Li A, Wang Q, He G, Jin J and Huang G: DEP domain containing 1 suppresses apoptosis via inhibition of A20 expression, which activates the nuclear factor $\mathrm{\kappa B}$ signaling pathway in HepG2 cells. Oncol Lett 16: 949-955, 2018.
16. Landi MT, Dracheva T, Rotunno M, Figueroa JD, Liu H, Dasgupta A, Mann FE, Fukuoka J, Hames M, Bergen AW, et al: Gene expression signature of cigarette smoking and its role in lung adenocarcinoma development and survival. PLoS One 3: e1651, 2008.

17. Huang W, Sherman BT and Lempicki RA: Systematic and integrative analysis of large gene lists using DAVID bioinformatics resources. Nat Protoc 4: 44-57, 2009.

18. Amin MB, Greene FL, Edge SB, Compton CC, Gershenwald JE, Brookland RK, Meyer L, Gress DM, Byrd DR and Winchester DP: The Eighth Edition AJCC Cancer Staging Manual: Continuing to build a bridge from a population-based to a more 'personalized'approach to cancer staging. CA Cancer J Clin 67: 93-99, 2017.

19. Liu Y,Zhang Y,ChiQ, Wang Z and Sun B: Methyltransferase-like 1 (METTL1) served as a tumor suppressor in colon cancer by activating 7-methyguanosine $\left(\mathrm{m}^{7} \mathrm{G}\right)$ regulated let-7e miRNA/HMGA2 axis. Life Sci 249: 117480, 2020.

20. Marinković M, Šprung M, Buljubašić M and Novak I: Autophagy Modulation in cancer: current knowledge on action and therapy. Oxid Med Cell Longev 2018: 8023821, 2018.

21. Manning BD and Toker A: AKT/PKB Signaling: Navigating the Network. Cell 169: 381-405, 2017.

22. Saxton RA and Sabatini DM: mTOR Signaling in Growth, Metabolism, and Disease. Cell 168: 960-976, 2017. Erratum in: Cell 169: 361-371, 2017.

This work is licensed under a Creative Commons Attribution-NonCommercial-NoDerivatives 4.0 International (CC BY-NC-ND 4.0) License. 